\title{
Uso de plantas medicinales en la prevención de enfermedades en Jalpa, Zacatecas
}

\section{Use of medicinal plants in disease prevention in Jalpa, Zacatecas}

\author{
GONZÁLEZ-GARCÍA, Arcelia†*, HERNÁNDEZ-SALAS, Claudia, MARTÍNEZ-ORTIZ, Rosa \\ María y TAVIZÓN-GARCÍA, Jesús Andrés
}

Universidad Autónoma de Zacatecas, Jardín Juárez 147, Zacatecas Centro, 98000 Zacatecas, Zacatecas, México.

\author{
ID 1 $1^{\text {er }}$ Autor: Arcelia, González-García / ORC ID: 0000-0003-0674-1072 \\ ID $1^{\text {er }}$ Coautor: Claudia, Hernández-Salas / ORC ID: 0000-0001-7492-1310 \\ ID $2^{\text {do }}$ Coautor: Rosa María, Martínez-Ortiz / ORC ID: 0000-0001-7811-169X \\ ID $3^{\text {er }}$ Coautor: Jesús Andrés, Tavizón-García / ORC ID: 0000-0003-2417-2571
}

DOI: $10.35429 / \mathrm{JOHS} .2020 .22 .7 .18 .25$

Recibido 10 de Enero, 2020; Aceptado 30 de Junio, 2020

\begin{abstract}
Resumen
La importancia en el uso de plantas medicinales se demuesta al cuantificar los suplementos alimenticios medicinales y dietéticos, utilizados en la práctica tradicional y empirica entre la población. Barthelson et al. (2006) (Acosta, 1993). Se estima que el $80 \%$ de la población mundial depende de remedios herbales tradicionales y que al menos 35,000 especies de plantas tienen potencial para uso medicinal. Annan y Houghton, (2007). Metodología y métodos: Es una investigación transversal, observacional y descriptiva. Se aplicó un cuestionario a 60 habitantes del Municipio de Jalpa, Zacatecas, que permitió comprender y reflexionar sobre el uso plantas medicinales como prevención de enfermedades. Se procesó la información a través del programa estadístico SPSS V. 24 y Excel. Resultados: Los entrevistados confirmaron el conocimiento de las plantas naturales, para uso medicinal fueron: el té de gordolobo, el temachaca, la flor de la alcaparra, azar. Las más utilizadas son: el Azar, Gordolobo, Temachaca, Flor de Alcaparra. Conclusiones: El uso de plantas medicinales siguen siendo una medicina alternativa, por un número considerable de población. Contribución: Los encuestados confirmaron el conocimiento en el uso de plantas como medicina natural.
\end{abstract}

Temachaca, Flor de alcaparra, Uso medicinal

\begin{abstract}
The importance in the use of medicinal plants is demonstrated when quantifying medicinal and dietary food supplements, used in traditional practice and empirically among the population. Barthelson et al. (2006) (Acosta, 1993). It is estimated that $80 \%$ of the world population depends on traditional herbal remedies and that at least 35,000 plant species have potential for medicinal use. Annan and Houghton, (2007). Methodology and methods: It is a transversal, observational and descriptive investigation. A questionnaire was applied to 60 inhabitants of the Municipality of Jalpa, Zacatecas, which allowed understanding and reflection on the use of medicinal plants as prevention of diseases. The information was processed through the statistical program SPSS V. 24 and Excel. Results: The interviewees confirmed the knowledge of natural plants, for medicinal use were: mullein tea, temachaca, caper flower, chance. The most used are: Azar, Gordolobo, Temachaca, Flor de Caper. Conclusions: The use of medicinal plants continues to be an alternative medicine, for a considerable number of the population. Contribution: Respondents confirmed knowledge of the use of plants as natural medicine.
\end{abstract}

Temachaca, Caper flower, Medicinal use

Citación: GONZÁLEZ-GARCÍA, Arcelia, HERNÁNDEZ-SALAS, Claudia, MARTÍNEZ-ORTIZ, Rosa María y TAVIZÓN-GARCÍA, Jesús Andrés. Uso de plantas medicinales en la prevención de enfermedades en Jalpa, Zacatecas. Revista de Ciencias de la Salud. 2020. 7-22:18-25.

\footnotetext{
*Correspondencia al Autor (Correo Electrónico: arcelia2009@live.com.mx)

$\dagger$ Investigador contribuyendo como primer Autor
} 


\section{Introducción}

Hoy en día se hace hincapié sobre la importancia en el uso de las plantas con fines terapéuticos, según las costumbres en la población del Municipio de Jalpa, Zacatecas, con frecuencia se adquieren en los mercados, su empleo se ha transmitido por generaciones, así mismo se destaca el conocimiento popular de diversas plantas medicinales y alimenticias entre los herbolarios, hierberos, yerberas o yerbateros, recomiendan el uso de plantas medicinales, los vendedores de los mercados, hacen promoción de las yerbas o plantas más utilizadas o recomendadas como: Árnica, Gordolobo, la Temachaca, entre otros. Las usan como consumo preventivo por su efectividad, curativa $\mathrm{y}$ alimenticia, además de tener un bajo costo. La Tamachaca es una planta nativa por uso medicinal y curativa, se podría decir al hacerle una cuantificación de la tabla nutrimental un "análisis proximal", constituyen los principales componentes de proteínas y los lípidos junto con los carbohidratos, (Nielsen, 1997).

El uso de medicinas alternativas como las plantas medicinales y los suplementos dietarios alimenticios ha sido una práctica tradicional que no ha caído en desuso Barthelson et al., (2006). Se estima que $80 \%$ de la población mundial depende de remedios herbolarios tradicionales y que al menos 35,000 especies vegetales presentan potencial para uso medicinal, Annan y Houghton, (2007): La gran diversidad vegetal y la amplia riqueza cultural de México han favorecido el aprovechamiento de las plantas con fines medicinales desde épocas prehispánicas (Martínez, 1996).

\section{Objetivo}

Identificar la frecuencia entre el uso y el conocimiento popular y científico de plantas naturales y medicinales más utilizadas en Jalpa, Zac.

\section{Justificación}

La importancia de llevar acabo esta investigación: es conocer la frecuencia y el conocimiento en el uso de consumo medicinal y preventivo de las plantas más conocidas: en el mercado de Jalpa, Zac, Aprovechando este recurso natural, dado las condiciones de cobertura de la salud, la medicina tradicional que aporta, este recurso natural.
Su composición nutrimental básica, de la temachaca para comprobar su eficacia nutracéutica, que se le ha conocido en forma natural. La temachaca es un Arbusto o árbol que mide hasta $14 \mathrm{~m}$ de alto. Nombre cienfífico: "Acasia coulteri Beth" Municipio: de Jalpa. Se considera una especie maderable pues se utiliza para la fabricación de postes para cercos. Sus hojas en racimos parecen plumas de ave. El cual se consume como tes o caldos, como comida tradicional en Jalpa, Zac. colinda al norte con el municipio de Huanusco al sur con Nochistlán y Apozol, al oriente con Huanusco y Calvillo, al poniente con Tepechitlán y Tlaltenango.

El nombre de "Jalpa" proviene del náhuatl xalli (en la arena), aunque en la época colonial se le conoció como "Santiago de Xalpa", ya comenzada la guerra de Independencia de México cambió a "Jalpa mineral" o "el mineral de Jalpa" por las minas que se encontraban en este municipio, al hacerse municipio se le denominó simplemente "municipio de Jalpa". Jalpa cuenta con una extensión de territorial de $733 \mathrm{~km}^{2}$ y con un porcentaje del $0.97 \%$ de la superficie del estado de Zacatecas. La población es de entre treinta mil habitantes, la mayoría está dentro de la ciudad y las comunidades circundantes. La población sigue creciendo desde que Jalpa se ha convertido en un importante centro de transporte entre las ciudades de Guadalajara, Aguascalientes y Zacatecas.

Un gran porcentaje de la población es mayor de 50 años; y hay significativamente más mujeres que hombres en el grupo de edad más joven; la mujer en general a comparación de los hombres es de aproximadamente $60 \%$ a $40 \%$.

Ésta desproporción se debe al hecho de que más hombres emigran a los Estados Unidos, $\mathrm{y}$ en este caso las mujeres suelen permanecer en el municipio con el resto de la familia. La población se incrementó un poco más con la llegada de los militares al municipio a causa del nuevo cuartel militar que se instaló en Jalpa, y desde allí varias personas de diferentes lugares han venido a establecerse en el municipio. 
Usualmente los comercios cercanos a la plaza principal (Mercado), cierran de 14:00 a 16:00 horas para ir a comer, dicha plaza es un punto de reunión y entretenimiento para personas de todas las edades, particularmente para población de mayor edad. Las familias jalpenses para abastecerse de agua cuentan con pozo propio para la extracción de agua o en caso contrario el agua que provee el municipio la almacenan en el "tinaco" (deposito de agua en el techo) o aljibe. La cocina Jalpense es meramente mestiza, sencilla y de carácter popular, está elaborada principalmente de carnes como: Chorizo, birria, carne adobada, longaniza, tacos de cabeza, enchiladas, menudo, pozole, condoches, tacos dorados, tacos de asada, tacazotas, caldo de temachaca, mezquitamal, atole gordo, cacahuatole, pinole, atole blanco, tacos de huache, cacahuate y semillas de calabaza con chile de árbol, tacos de mole de codorniz.

Queso de tuna, dulce de biznaga, dulce de leche, dulce de camote, alfajor de coco, dulce de calabaza, ate de membrillo, garampiñados, ate de guayaba, capirotada, torrejas, obleas con cajeta de leche, greñudas, charrascas, guayabate, dulce de tuna, dulce de chilacayote, melcochas de semana santa, nieves de garrafa ( sabores de limón, vainilla, piña, endiablados.En nuestro país las plantas medicinales no están validadas científicamente y se "desconoce si son seguras o eficaces "por lo que, como todo medicamento, debe ser empleado con cautela, ya que puedan aparecer cuadros tóxicos derivados del consumo de plantas":

El nombre científico de la alcaparra es: Capparis spinosa. También en la actualidad se utiliza en gastronomía y en otras regiones, la alcaparra es un arbusto semileñoso, perenne muy ramificado. Su altura naturalmente no supera el medio metro, pero puedo llegar 1,5 metros se usa como valla natural en jardines, desarrolla espinas hasta $1 \mathrm{~cm}$ de largo: al adquirirla retirar los capullos comestibles, para su consumo humano el cultivo de la alcaparra, además la planta es resistente a las plagas, heladas, granizos etc.
La floración se produce a partir del mes de mayo: Aunque se aprovecha los capullos conocidos como las alcaparras, también se consumen los frutos llamados alcaparrones: una de las maneras para su preparación es curtidos de vinagre y sal, con sabor amargo picantes pero son tónicas y dan apetito contiene algunos componentes activos como pectina y saponinas y sales, debido a la cual se le atribuye propiedades medicinales, que van desde un carácter diurético, expectorante contra la fragilidad capilar hasta antihemorroidal, contra las aftas bucales mediante la su decocción) sus flores llamativas con sus pétalos blancos o rosáceos y unos estambres muy largos, con anteras de color violeta, axilares que nacen de la misma intersección de hojas con el tallo. Y como sus flores llamativas, la flor de alcaparra arbusto perenne de raíz leñosa, crece en lugares de clima cálidos soleados.

El término de la alcaparra proviene del "griego caparis y del árabe alkabara" también conocida como la "tapenera" apareciendo como referencia, textos antiguos sobre propiedades curativas gastronómicas de la planta. Una de las propiedades principales de las alcaparras y alcaparrones son el agua, así como los hidratos de carbono. El botón de la flor del alcaparro la alcaparra o tápena, es valorado por sus aplicaciones en la farmacopea gracias a sus propiedades diuréticas, antirreumáticas, antiartríticas debido a la capirituna y cosmetológica, también es utilizada en la provincia de Zacatecas para el malestar de muelas, mediante enjuagues bucales, curación de heridas infectadas, mediante infusiones que combaten a padecimientos estomacales, evitación de la caída de cabello.

Las alcaparras: la raíz de las fojas y el fruto son calientes abrideras y disolutivas alivian los tumores de estómago así como abre los caños del hígado (conductos viliares) y del baso menstruación de la mujer les da apetito de comer: poseen propiedades curativas, por distintas causas pueden transformarse en factores de intoxicación. Esto no quiere decir que toda la fitoterapia sea nociva, muchos medicamentos proceden de plantas, pero el conocimiento de las hierbas curativas muchas veces es limitado y se desconocen sus efectos, lo que hace necesario informar y alertar sobre su correcta prevención y administración y prescripción. 
El uso de plantas ó hierbas con fines medicinales es un hábito ancestral Martínez, (2008), desconociéndose en varias casos que debe ser controlado. Si bien estas infusión o tés pueden poseer propiedades curativas, por distintas causas pueden presentar factores de intoxicación. Siendo de infusión y de cocción, algunas alcanzan una gran concentración de sus sustancias activas, por lo tanto, es impredecible conocer con exactitud la cantidad de hierba a usar, en una densidad de agua y el tiempo de cocción. Ya sea ideal súper valoración de lo natural o por la situación precaria de pobreza de grandes sectores de la población, hacer uso de esas plantas para curarse de diversos malestares, hoy en día es una medida que ha ido en aumento en los últimos tiempos. En nuestros países, las plantas medicinales prácticamente no están validadas científicamente y "se desconoce si son seguras y eficaces" como todo medicamento, deben ser empleadas con cautela. (Rizzo, 2000). Abundantes hierbas de plantas medicinales son vendidas en farmacias y herboristerías, inclusive sin la debida autorización de las autoridades correspondientes e ignorándose además lo que ocurre con la automedicación de las mismas (Medina, 1999).

Si bien la utilización de plantas, hierbas medicinales curativas es una medicina alternativa válida, en algunas oportunidades su uso es empírico, y hasta fraudulento. Pueden aparecer cuadros tóxicos derivados del consumo de plantas. Niños, desde el nacimiento, ancianos, mujeres en gestación o lactancia, son los individuos más propensos. Existen tipos de intoxicaciones agudas, las cuales son fácilmente detectables, por los síntomas de la intoxicación, que aparecen en el corto tiempo después de su administración e intoxicaciones crónicas, no son fácilmente detectables, resultando difícil atribuir la presencia de algún trastorno de la salud por consumo de alguna planta medicinal, dado que el individuo se relaciona en forma permanente con su medio.

Se puede considerar además que las plantas medicinales pueden presentar dos tipos de toxicidad: a) Toxicidad intrínseca, en la cual la planta produce metabolitos tóxicos para el ser humano. La presencia de síntomas dependerá de la dosis (intoxicación aguda) o del tiempo de empleo (intoxicación crónica).
Ejemplos de metabolitos tóxicos: alcaloides, algunos flavonoides, glicósidos cardiotónicos y cianogenéticos, ác. aristolóquicos, derivados terpenoides (taxol, ascaridol, cucurbitacinas). b) Toxicidad extrínseca, la planta no produce sustancias potencialmente tóxicas. Laxicidad proviene de la presencia de contaminantes (mezclas con especies tóxicas, metales pesados, pesticidas de la agricultura, micotoxinas, alta carga microbiana): se determina la existencia de plantas con uso medicinal que en otros lugares se vuelven tóxicos. Es por ello que se vuelve trascendente estudiar la composición fitoquímica. (Ricciardi, 2001).

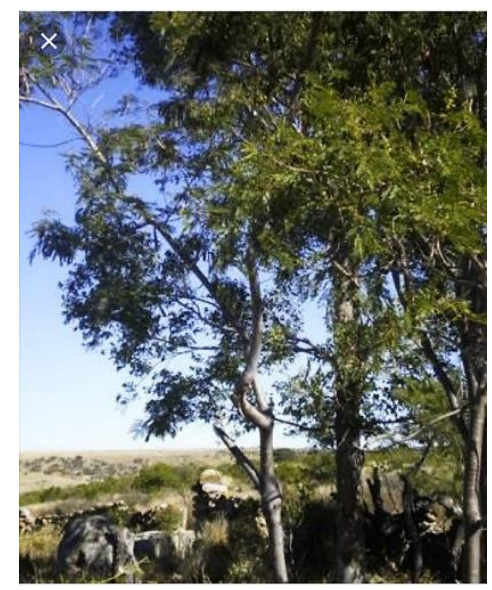

Figura $1 \mathrm{El}$ árbol de al temachaca Fuente: Elaboración propia

\section{Metodología y Métodos}

Se realizó una investigación transeccional, observacional, y descriptiva acerca de los usos costumbres con el conocimiento plantas medicinales y el uso prevención de ambas, de la flor de alcaparra como la temachaca con el fin de contribuir un buen manejo para sus usos y beneficios y riesgos, obtenida mediante datos registrados, de casos más frecuentes para su consumo de plantas, en la cual se tomó una muestra aleatoria de 60 personas encuestadas ambos sexos de edades de 20 a 60 años. 
Se realizó una investigación donde: la prevalencia de los porcentajes, entre estas dos plantas vendidas a comparación de la temachaca y flor de alcaparra haciendo hincapié que tiene un al contenido proteico, en proteínas que nos ayuda al sistema inmunológico, asi como anemías hemorragias en el ser humano, esta investigación se llevó acabo de tipo transversal longitudinal de una observación teórica-metodológica que nos permite comprender y reflexionar observar los procesos, prevención para explicar cómo la independencia específico.

Objetivo: Identificar cual es el uso y conocimiento popular de las plantas medicinales para la prevención de ser consumidas, que conlleva al razonamiento natural, producido por investigaciones fitoquímicas y/o clínicas. Se realizaron técnicas cualitativas para rescatar serie datos opiniones, creencias, usos, de conceptos, valores de proceder a obtener respuestas acerca del proceso de la práctica médica herbolaria. Se realizó un cuestionario semiestructurado y semidirigido. (Babbie, 1988) que ofreció la ventaja de los informantes respondieron con temáticas similares, de manera que el análisis de los datos se manejó bajo una misma pauta. Cuestionario de porcentajes aplicados de la frecuencia de Flor de alcaparra y temachaca los porcentajes más relevantes. ¿Conoce usted algunas plantas
medicinales?

- ¿Cuáles son las plantas medicinales que consumen con más frecuencias aquí ?

- ¿Algunas veces ha tomado estas plantas como alimentos o infusión de te?

- ¿ $\quad$ Sabe usted que la planta de la temachaca es de uso medicinal ?

- ¿A escuchado usted que la planta de flor de alcaparra es para uso medicinal ?

- ¿Con que frecuencia consume la flor de alcaparra ?

- ¿ Conoce el tiempo en que se da la temachaca?

- ¿Conoce el tiempo en que se da la flor de alcaparra ?
- ¿Recomendaría la alcaparra como uso preventivo para de salud?

¿Recomendaría tomar el uso de la preparación de la tema chaca como preventivo de la salud?

Los entrevistados confirmaron el conocimiento que por generaciones han tenido en el uso de las plantas de la medicina natural (ver Tabla 1). Donde se presentan los porcentajes de las plantas más recomendadas y conocidas para su uso medicinal y preventivo.

\begin{tabular}{|l|r|r|r|}
\hline $\begin{array}{l}\text { Nombre de } \\
\text { las plantas }\end{array}$ & $\begin{array}{c}\text { \% de } \\
\text { conocimiento de } \\
\text { uso medicinal }\end{array}$ & $\begin{array}{c}\text { \% de } \\
\text { personas } \\
\text { que lo } \\
\text { conocen }\end{array}$ & $\begin{array}{c}\text { \% de } \\
\text { encuestados } \\
\text { que conocen } \\
\text { otras } \\
\text { plantas }\end{array}$ \\
\hline $\begin{array}{l}\text { Flor de } \\
\text { alcaparra }\end{array}$ & 33.33 & 25 & 16.66 \\
\hline Temachaca & & & \\
\hline Te de azar & 20 & 8.33 & \\
\hline
\end{tabular}

Tabla 1 Conocimiento y frecuencia del uso de tres principales plantas medicinales utilizadas

Fuente: 60 habitantes encuestados de Jalpa, Zac.

\section{Frecuencia en el uso de las tres plantas medicinales.}

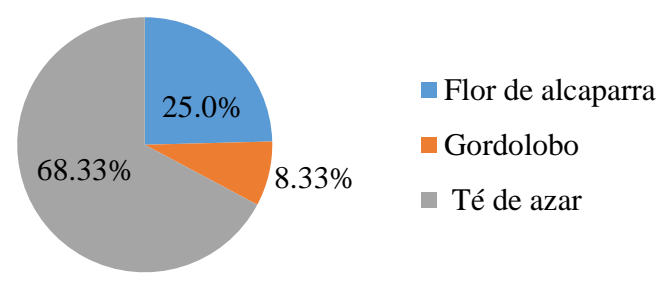

Gráfico 1 Frecuencia en el Uso de las tres plantas medicinales

Fuente: 60 personas encuestadas en el Mercado de Jalpa, Zac

El principal mercado del Municipio de Jalpa Zacatecas, como uno de los puntos primordiales de distribución a los comunidades que la compran para su alimentación del estado, el recurso de consumo (Herrera, 1992), además de que representan una fuente de información y un espacio social de conocimientos de enseñanzas y saberes y usos tradicionales sobre plantas medicinales (Martínez Moreno et al., 2006: 79-80. 


\section{Resultados y Conclusiones}

De los resultados obtenidos, de la primera pregunta es saber en primera instancia si se tiene conocimiento de plantas medicinales, para ello se consideró una muestra aleatoria de 60 personas que acudieron a la plaza al mercado de Jalpa Zac, de las cuales se manifestó solo el $16.66 \%$ contestaron que sí, $1.66 \%$ no conoce ninguna, el $81.66 \%$ desconocen plantas pero no precisamente las de uso medicinal.

De las plantas de uso común que tienen conocimiento fueron las siguientes:

\begin{tabular}{|l|rr|}
\hline \multicolumn{2}{|c|}{$\begin{array}{c}\text { Nombre de la } \\
\text { planta }\end{array}$} & $\begin{array}{c}\text { \% de personas que la } \\
\text { conocen }\end{array}$ \\
\hline Flor de alcaparra & 25 \\
\hline Gordolobo & 8.3 \\
\hline Té de azar & & 68.3 \\
\hline
\end{tabular}

Tabla 2 Prevalencia de uso común de las plantas Fuente: 60 personas encuestadas en el Mercado de Jalpa, Zac.

Referente a si ha consumido plantas como alimentos o infusión de te es importante mencionar que un alto porcentaje (66.66 \%) utilizó la Temachaca, un $8.33 \%$ menciona el gordolobo y el resto mencionó otras plantas (25 $\%)$.

Al ir focalizando la investigación hacia dos de las plantas de uso medicinal como son la Temachaca y Flor de alcaparra, el gráfico que a continuación se presenta nos muestra los resultados obtenidos:

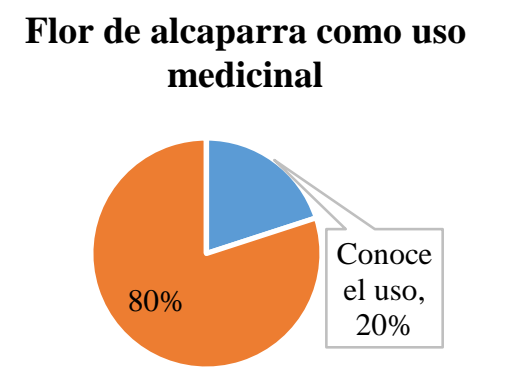

- Conoce el uso $\quad$ Desconoce el uso

Gráfico 2 Frecuencia en el uso de Flor de Alcaparra

Fuente: 60 personas encuestadas

\section{Temachaca como uso medicinal}

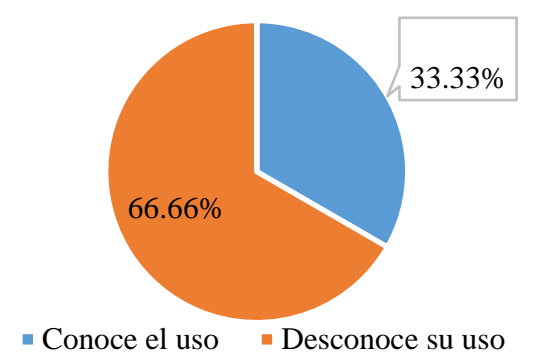

Gráfico 3 Frecuencia en el uso de Temachaca Fuente: 60 personas encuestadas

Es importante mencionar que particularmente para el consumo de la Flor de alcaparra 10 de las personas encuestadas lo hacen a diario, 9 de ellas por semana, 15 esporádicamente y el resto no la consume.

Referente al tiempo de cultivo de las dos plantas antes mencionadas, particularmente para la Temachaca el $41.66 \%$ sabe la temporada en la que se puede encontrar, $13.33 \%$ no lo sabe y el resto (27 personas) $45 \%$ conoce pero el de otras plantas. Por su parte, en la Flor de alcaparra solo el $13.33 \%$ ( 8 personas) conoce el período en el que se puede obtener.

Finalmente se hace mención de la recomendación de estas dos plantas para su uso preventivo en la salud y las respuestas que se encontraron son las siguientes:

\begin{tabular}{|l|r|l|r|}
\hline \multicolumn{2}{|c|}{ Temachaca } & \multicolumn{3}{c|}{ Flor de alcaparra } \\
\hline Recomiendan & $91.66 \%$ & Recomiendan & $33.33 \%$ \\
\hline No la recomiendan & $3.33 \%$ & No la recomiendan & $25 \%$ \\
\hline Recomiendan otras & $5 \%$ & Recomiendan otras & $41.66 \%$ \\
\hline
\end{tabular}

Tabla 3 Recomiendan el uso de las plantas Temachaca y Flor de alcaparra

Fuente: 60 personas encuestadas en el Mercado Jalpa, Za 


\section{Discusión}

En base a las recomendaciones y conocimiento de la demanda del consumo de plantas y la frecuencia de ser más vendida y más comercializada por su debido costo bajo económico como la temachaca y la flor de alcaparra, ya que presentan un contenido proteico, ayuda al sistema inmunológico previene hemorragia, la flor de alcaparra como condimento, infusiones, enjuagues bucales. el gordolobo, ya que es empleado para resfriados y tos bronquitis y diarrea. (La Marinoff. et al) como el té de azar, debido al contenido de sus propiedades fitoterápicos, como la flor de alcaparra puede presentar, intoxicaciones crónicas con el uso prolongado, principales síntomas es la anemia que produce. (Gurni, 2000), (Usandizaga, 2000), ya que las plantas medicinales sufren modificaciones cuantitativas, debido a los factores, como cambios climáticos. Se hace un análisis de los resultados de las plantas naturales conocidas en la sociedad de ser consumidas arrojados de cada una de ellas, los cuales fueron registrados en las tablas correspondientes. Lo significativo en este estudio, a partir de que la consumen la saborean en platillos de degustación asi como, también su enriquecimiento proteico de proteínas juegan un papel importante en la salud bucal de sus hijos, ya que ellos son sujetos que trasmiten microrganismo a la cavidad bucal de los infantes provocando una colonización y cambio en su flora bacteriana normal. Por lo tanto es importante mencionar que las plantas medicinales mencionadas como vendidas con más frecuencia son: el te de azar, Gordolobo, la temachaca, la flor de alcaparra, entre otras. "La ingesta de Temachaca nos ayuda al sistema inmunológico por su alto contenido de proteínas, como energizante, además nos sirve para anemias, hemorragias, para combatir el estreñimiento, eficaz como potencial energético, la flor de alcaparra se usa: como condimento para comidas, enjuajes bucales, para dolores de menstruación, pero si no se tiene cuidado, el niño puede terminar deshidratado; su alta concentración, que lo hace tóxico, siendo el principal motivo de intoxicación en niños, ambas han registrado casos letales.
El gordolobo se emplea en tos, bronquitis diarrea: La Marinoff et al: para los fitoterápicos de la flor de alcaparra es una planta particularmente infusiones Fito terapéuticas, se trata de buen antirreumático, pero también tiene compuestos que producen intoxicación crónica, uno de los primeros síntomas es el decaimiento debido a la anemia que produce. (Gurni, 2000 (Usandizaga, 2000). Las plantas medicinales sufren modificaciones cuantitativas en sus constituyentes principales originados por efectos climáticos o de crecimiento de la misma, el contenido de ciertas sustancias varía cuando la planta alcanza su óptimo desarrollo.

En ocasiones, algunos que se entrevistaron, presentaron algunos resistencia al ser cuestionados, así mismo consideran los venderes que las personas acude a este tipo de terapéutica, entre otras cuestiones, porque presenta un costo más económico el hacer hacer uso de estas plantas de medicinales que la medicina de patente. Al cuestionar a los informantes por qué las plantas medicinales curan, contestaron que este tipo de terapia esta vigente en la actualidad, lo que concuerda con Ryesky (1976).

\section{Agradecimiento}

Los autores agradecen a los revisores anónimos y al editor sus valiosas sugerencias para mejorar el documento, así como a los informantes por su participación, el proceso de enfermedad, consumo y/o curación es congruente a su salud y a lo tradicional ó cultural.

\section{Conclusiones}

El uso de plantas medicinales siguen siendo una medicina alternativa, para un número considerable de población, de ninguna manera pueden ser sustitutos de la medicina científica, y se debe utilizar con precaución siguiendo los protocolos basados en métodos cienfíficos. Se deben aumentar el tamaño de la muestra para tener una mayor representatividad. 


\section{Referencias}

Acosta D.L.L, Lérida L., (1993), Proporciónese Salud: cultive plantas medicinales, Editorial Científico Técnica, La Habana. Aguirre Beltrán, Gonzalo, 1986, Antropología médica, Centro de Investigaciones y Estudios Superiores en Antropología Social, México. AguirreHernández, Eva et al.,

Aguirre-Hernández $\quad \mathrm{E}^{1}$, Martínez $\quad$ AL, GonzálezTrujano ME, Moreno J, Vibrans H, Soto-Hernández M. (2007). "Pharmacological Evaluation of the Anxiolytic and Sedative Effects of Tilia americana L. var. Mexicana in Mice", en Journal of Ethnopharmacology, vol. 109, núm. 1, pp. 140-145.

Babbie, E.R., (1988), Métodos de investigación por encuesta, Biblioteca de la Salud, Fondo de Cultura Económica, México

Barragán S. A, (2006), "La práctica de la autoatención por fitoterapia en un grupo de familias mexicanas", en Archivos en Medicina Familiar, vol. 8, pp. 155-162.

Bodeker, G, 1994, "Savoir Tradicional et Politique de Santé Publique", en Nature et Ressources, vol. II, núm. 30, pp. 5-16. Borgatti, Stephen Peter, 1992, Anthropac 4.0 Reference Manual, Analytic Technologies, Columbia University, Columbia.

Borgatti, S. P, 1992, Anthropac 4.0 Reference Manual, Analytic Technologies, Columbia University, Columbia.

Browner, C- H., Ortiz de M.B y J. Rubel A, 1992, "El análisis comparativo de sistemas médicos", en Paola Sesia (ed.), Medicina tradicional, herbolaria y salud comunitaria en Oaxaca, Gobierno.

Browner, C H., Bernard O. de M- J. Rubel A.(1992), "El análisis comparativo de sistemas médicos", en Paola Sesia (ed.), Medicina tradicional, herbolaria y salud comunitaria en Oaxaca, Gobierno del Estado de Oaxaca, Centro de Investigaciones y Estudios Superiores en Antropología Social, Oaxaca, pp. 223-263.
Browner, C. H., Ortiz de M.B, J .A R, (1992), "El análisis comparativo de sistemas médicos", en Paola Sesia (ed.), Medicina tradicional, herbolaria y salud comunitaria en Oaxaca, Gobierno del Estado de Oaxaca, Centro de Investigaciones y Estudios Superiores en Antropología Social, Oaxaca, pp. 223-263.

Bye, R y Linares E, (1987), "Usos pasados y presentes de algunas plantas medicinales encontradas en los mercados mexicanos", en América Indígena, vol. 47, núm. 2, pp. 200-230.

Campos-B, P. et al., (2005), "Effect of Gnaphalium conoideum HBK on Guinea Pig Airway Smooth Muscle: Role of L-type Ca2 Channels", en Journal of Ethnopharmacology, vol. 97, núm. 2, pp. 267-272.

Campos N, R, (1993). "Estudios urbanos en México sobre el uso de las plantas medicinales", en Jesús Kumate (comp.), La investigación científica de la herbolaria medicinal mexicana, Secretaría de Salud, México

Casais M.C, R., R.A. L.C, Casais M.C, Técnicas de Separación en Química Analítica, Ed. Síntesis, Madrid.

Kumate J. (2005). (comp.), La investigación científica de la herbolaria medicinal mexicana, Secretaría de Salud, México.

Matissek y col, Análisis de los Alimentos. Fundamentos, Métodos y Aplicaciones, Ed. Acribia. -

Pavia D.L, G.M. Lampman, G.S. K, (1978). Química Orgánica Experimental, Ed. Universitaria de Barcelona Eunibar, 1978. 